\title{
The application of conventional us and transthoracic ultrasound elastography in evaluating peripheral pulmonary lesions
}

\author{
HONG WEI, YUCHAN LU, QIAO JI, HANG ZHOU and XIANLI ZHOU \\ In-Patient Ultrasound Department, The Second Affiliated Hospital of Harbin Medical University, \\ Harbin, Heilongjiang 150086, P.R. China
}

Received October 19, 2017; Accepted June 6, 2018

DOI: $10.3892 /$ etm.2018.6335

\begin{abstract}
The aim of the present study was to evaluate the diagnostic value of ultrasound (US) elastography in differentiating between benign and malignant peripheral lung lesions (PLLs). This retrospective study included 91 consecutive patients with 91 PLLs. Conventional US, strain elastography (SE), acoustic radiation force impulse imaging (ARFIimaging) and point share wave elastography ( $p-S W E$ ) were performed. All of the pathological results were confirmed by US-guided biopsies or surgeries. There were 36 benign PLLs and 55 malignant PLLs on pathology. For conventional US, a lesion diameter $\geq 5 \mathrm{~cm}$, irregular contour, presence of air bronchogram and non-abundant vascularity were predictive factors of malignancy $(\mathrm{P}<0.05)$. SE scores were observed to be invalid in differentiating between malignant and benign PLLs $(\mathrm{P}=0.542)$. For ARFIimaging scores, an elasticity score of 3 or greater was predictive of malignancy, with a sensitivity of $83.6 \%(46 / 55)$ and a specificity of 52.8\% (19/36). For p-SWE, the share wave velocity of malignant PLLs was higher than benign ones $(2.47 \pm 0.92$ vs. $1.85 \pm 0.92 \mathrm{~m} / \mathrm{sec} ; \mathrm{P}=0.0022)$. When $1.951 \mathrm{~m} / \mathrm{sec}$ was selected as the cut-off value, a sensitivity of $70.9 \%(39 / 55)$ and a specificity of $69.4 \%(25 / 36)$ were obtained. Thus, US, particularly US elastography, is helpful in distinguishing malignant PLLs from benign PLLs.
\end{abstract}

\section{Introduction}

In recent years, lung cancer has become one of the most common malignant tumors threatening human health (1), and its incidence rate has been on the rise $(2,3)$. Therefore, it is imperative to know how to accurately distinguish malignant lung lesions (MLLs) from benign lung lesions (BLLs).

Correspondence to: Professor Xianli Zhou, In-Patient Ultrasound Department, The Second Affiliated Hospital of Harbin Medical University, 246 Xuefu Road, Nangang, Harbin, Heilongjiang 150086, P.R. China

E-mail: zhouxianli@ems.hrbmu.edu.cn

Key words: ultrasound elastography, peripheral lung lesions, ultrasonography, lung
Young women, pregnant women, and critically ill patients cannot tolerate chest X-rays and computed tomography (CT) because of radiation, although they are first-line imaging modalities for assessing the morphological features of pulmonary lesions (PLs) (4-6). When a PLL is present and space-occupied, the acoustic windows of the lung tissue are available, which makes the ultrasonic wave pass. Hence, after selecting the appropriate detection angles for PLLs below the pleura, optimal images can be obtained during transthoracic ultrasound (TUS) examination $(7,8)$. The advantages of US compared to X-ray or CT are its easy operation, lower cost, and lack of exposure to radiation. In addition, US-guided biopsy is as accurate as CT-guided biopsy for the diagnosis of PLLs or pleural lesions. Real-time guidance can be performed using US, making the procedure faster and free of ionizing radiation (9).

In recent years, US elastography has been widely applied to the differential diagnosis of thyroid, breast, liver, and prostate lesions (10-13). It can reflect the intrinsic features of the lesions. The tissue stiffness of malignant lesions is usually harder than those that are benign $(14,15)$. US elastography is mainly categorized as strain elastography (SE) and shear wave elastography (SWE), in which the information about the tissue stiffness can be obtained by pressure or shear force that deforms the tissue directly or indirectly (16). The tissue stiffness obtained by manual compression can be affected by different operators, leading to inter-observer differences. However, acoustic radiation force impulse (ARFI) elastography that includes an ARFI imaging mode and a point share wave elastography ( $\mathrm{p}$-SWE) mode can conduct qualitative or quantitative measurement objectively by assessing the elastogram in grayscale or the shear wave velocity (SWV) value of the lesions (17).

Sperandeo et al (18) reported that lung SE enables good non-invasive imaging of PLs, as it provides information on their stiffness and improves the accuracy and yield of fine needle aspiration biopsy (FNAB). But there are few reports about the usefulness of ARFI elastography in diagnosing PLLs. As such, this study purported to assess the value of TUS elastography in distinguishing malignant from benign PLLs.

\section{Materials and methods}

Study population. This retrospective study was approved by the Ethics Committee of this tertiary hospital (the Second 
Affiliated Hospital of Harbin Medical University, Harbin, China) and the written informed consent of patients was obtained. From January 2013 to January 2015, 201 consecutive patients with PLLs found by chest X-ray or CT examination were enrolled in this institution (the Second Affiliated Hospital of Harbin Medical University). The exclusion criteria were as follows: i) Trouble breathing $(\mathrm{n}=20)$; ii) the lesions were covered by bone tissue $(\mathrm{n}=15)$; iii) no pathological result $(\mathrm{n}=42)$; and iv) accompanied with pleural effusion ( $\mathrm{n}=33$ ). If there were multiple lesions, the largest was incorporated into the study. Finally, conventional US and elastography examinations (including SE and ARFI elastography) were performed on the remaining 91 patients. The final pathological results were obtained by surgery or biopsy.

Conventional US, SE, ARFIimaging, and p-SWE. Conventional US and US elastography examinations were performed by a radiologist with at least 10 years of experience (H.W.) in ultrasonic examination with the US machine (Siemens Acuson S2000; Siemens Medical Solutions, Eschborn, Germany) and the 4C1 convex vibration probe (frequency range, 1.5-4.0 MHz). The focus and gain of images were adjusted to obtain optimal images. The probe was held perpendicularly to the skin surface on the intercostal space and parallel to the ribs throughout the examinational process. Using a 2-dimensional US, the PL was assessed for its size, position within the lobe, shape, margin, internal echogenicity, echotexture, and presence of air bronchogram. Then the color Doppler US mode was applied and the gain was adjusted to the optimal level so background color signals did not display any noise throughout the process. Afterwards, SE was applied with mild manual compression. The region of interest (ROI) box was aimed at the whole lesion and some peripheral normal tissues. The radiologist kept the probe motionless for $5 \mathrm{sec}$ to obtain optimal elastic images. The US image was on the left side of the screen while the SE image was on the right side and color-coded. ARFI imaging was performed after SE. The ROI box was placed in the same position as SE. The ARFI image was on the right side of the screen and gray-coded. After that, the p-SWE mode was initiated. A $6 \times 5 \mathrm{~mm}$ ROI box was placed at the solid portion without air covering the lesion. The SWV value was displayed on the right side of the screen. Seven consecutive measurements were performed at the same depth of each lesion. Finally, the average value was obtained after removing the maximum and minimum values.

US-guided core needle biopsy. Before US-guided core needle biopsy (US-CNB), all patients signed the informed consent forms. The procedure was performed by 2 experienced radiologists (Q.J. and H.W.) using a US machine (Philips iU22; Philips Ultrasound, Inc., Reedsville, PA, USA), a Bard automatic biopsy gun, and a biopsy needle (16 G; Bard Peripheral Vascular, Inc., Tempe, AZ, USA). Tissue samples were taken under the guidance of US. Biopsy was repeated 3-4 times according to the specimen quality. Afterward, pathological diagnosis was performed by 1 pathologist with 20 years' work experience who was blinded to previous US findings.

Image interpretation. The images were interpreted by 2 investigators (H.W. and Y.L.) who were blinded to the pathological results. The size of the lesions was defined by the longest diameter measured on US, with $<5 \mathrm{~cm}$ and $\geq 5 \mathrm{~cm}$. The shape of the lesions was defined as having regular contours (triangular or rounded) and irregular contours (non-triangular or non-rounded). The margin was defined as smooth (at least 50\% of the lesion was visible) and rough (at least $50 \%$ of the lesion was invisible). The internal echogenicity of the lesions was defined as homogeneous hyperechoic/isoechoic/hypoechoic/anechoic or heterogeneous hyperechoic/isoechoic/hypoechoic/anechoic. The lesions were grouped as with or without air bronchogram (punctiform or linear hyperechoic artifacts within the lesions) (19). The vascularity of the lesions was classified into abundant ( $\geq 2$ blood vessel signals) and non-abundant ( $<2$ blood vessel signals). As current international recommendations do not yet provide a specific way to classify the stiffness of PLL, we customized the classifications. SE imaging was described by the color scale of the ROI, with red, green, and blue delineating from soft to hard, and SE was classified into 4 grades. ARFI imaging was classified into 4 grades from soft to hard according to the grayscale (Table I).

Hematoxylin and eosin $(H \& E)$ staining. Lung tissues were fixed in formalin, cleared in xylene (both from Sigma-Aldrich; Merck KGaA, Darmstadt, Germany) and embedded in paraffin. The paraffin-embedded lung tissue samples were examined by conventional light microscopic examination: $5 \mu \mathrm{m}$ sections were stained with H\&E, and assessed in a blinded manner. An automatic microscope (Provis AX-70) with a camera (Olympus Corporation, Tokyo, Japan) was used to capture the microscopic images of the lung samples. The morphometric analysis was performed using ImageJ software version 1.60 (National Institutes of Health, Bethesda, MD, USA).

Statistical analysis. All statistical analyses were carried out using SAS 9.13 statistical software (SAS Institute Inc., Shanghai, China). The geometric mean and standard deviation were used to describe measurement data and compared between groups using Student's t-test. Frequency and percentage were used to describe counting data and compared between groups using the Chi-square test or Fisher's exact test. A receiver operating characteristic (ROC) curve was created to analyze the accuracy of each variable. $\mathrm{P}<0.05$ was considered statistically significant.

\section{Results}

This study involved 50 males and 41 females, with an average age of 55.11 \pm 11.11 years (range, 24-86 years). The mean diameters of the BLLs and MLLs were 58.17 \pm 20.18 and $72.05 \pm 24.27 \mathrm{~mm}$, respectively (range, 20.1-141.4 mm). The pathological results were confirmed by US-CNB procedures in 80 patients and by surgery in 11 patients. There were 36 benign lesions ( 32 chronic inflammatory lesions, 4 tuberculosis) and 55 malignant lesions (33 adenocarcinomas, 21 squamous cell carcinomas (Fig. 1), and 1 small cell lung cancer). Female sex $(\mathrm{P}=0.009)$ and older age $(\mathrm{P}=0.002)$ were predictive of malignancy. The location of the lesions and smoking history were not relevant factors in this study ( $\mathrm{P}>0.05$; Table II).

Conventional US features. On conventional US, characteristics such as a lesion diameter $\geq 5 \mathrm{~cm}$, irregular contours, 
Table I. Scoring systems for strain elastography imaging and acoustic radiation force impulse imaging in the peripheral pulmonary lesions.

\begin{tabular}{lll}
\hline Score & \multicolumn{1}{c}{ SE imaging } & ARFI-imaging \\
\hline 1 & Shown homogeneously in red and green & Displayed $<25 \%$ areas of dark gray or black \\
2 & Shown predominantly in green, a few blue areas or spots & Displayed 25-50\% areas of dark gray or black \\
3 & Shown predominantly in green, or with equal areas in blue & Displayed 50-75\% areas of dark gray or black \\
4 & Shown predominantly in blue with or without a few green areas & Displayed $>75 \%$ areas of dark gray or black \\
\hline
\end{tabular}

SE, strain elastography; ARFI, acoustic radiation force impulse.

Table II. Basic characteristics of patients with peripheral pulmonary lesions.

\begin{tabular}{lccc}
\hline $\begin{array}{l}\text { Basic } \\
\text { characteristic }\end{array}$ & $\begin{array}{c}\text { Benign, } \\
\mathrm{n}(\%)\end{array}$ & $\begin{array}{c}\text { Malignant, } \\
\mathrm{n}(\%)\end{array}$ & P-value \\
\hline $\begin{array}{l}\text { Sex } \\
\text { Female }\end{array}$ & $10(24.4)$ & $31(75.6)$ & $0.009^{\mathrm{a}}$ \\
$\quad$ Male & $26(52.0)$ & $24(48.0)$ & \\
Age (years) & & & \\
$<50$ & $10(71.4)$ & $4(28.6)$ & $0.002^{\mathrm{a}}$ \\
$50-69$ & $24(40.0)$ & $36(60.0)$ & \\
$\geq 70$ & $2(11.8)$ & $15(88.2)$ & \\
Smoking history & & & \\
$\quad$ No & $10(37.0)$ & $17(63.0)$ & 0.749 \\
Yes & $26(40.6)$ & $38(59.4)$ & \\
Location (in lung) & & & \\
Right & $18(35.3)$ & $33(64.7)$ & 0.348 \\
Left & $17(42.5)$ & $23(57.5)$ & \\
\hline
\end{tabular}

${ }^{\mathrm{a}} \mathrm{P}<0.01$.

presence of air bronchogram, and non-abundant vascularity showed significant differences between benign and malignant lesions ( $\mathrm{P}<0.05$; Table III). In addition, using $5 \mathrm{~cm}$ as a cut-off value for the lesion diameter led to a sensitivity of $87.3 \%$ and a specificity of $38.9 \%$. The margin and echogenicity of lesions presented no significant differences $(\mathrm{P}>0.05)$.

US elastography features. For elastography features (Table IV), no significant difference was found in SE between BLLs and MLLs $(\mathrm{P}=0.542)$. But significant differences were found in ARFI imaging and p-SWE between BLLs and MLLs $(\mathrm{P}<0.05)$. On ARFI imaging scores, the lesions with 1-2 and 3-4 were classified as benign and malignant, respectively, and corresponding sensitivity, specificity, positive predictive value (PPV), and negative predictive value (NPV) were $83.6 \%$ (46/55), 52.8\% (19/36), 73.0\% (46/63), and $67.9 \%$ (19/28), respectively. Using this classification method, 9 false negative lesions and 17 false positive lesions were found. The SWV values of the MLLs were higher than those of the BLLs $(2.47 \pm 0.92$ vs. $1.85 \pm 0.92 \mathrm{~m} / \mathrm{sec} ; \mathrm{P}=0.0022)$. According to the ROC curve, the area under the curve (AUC) of p-SWE was
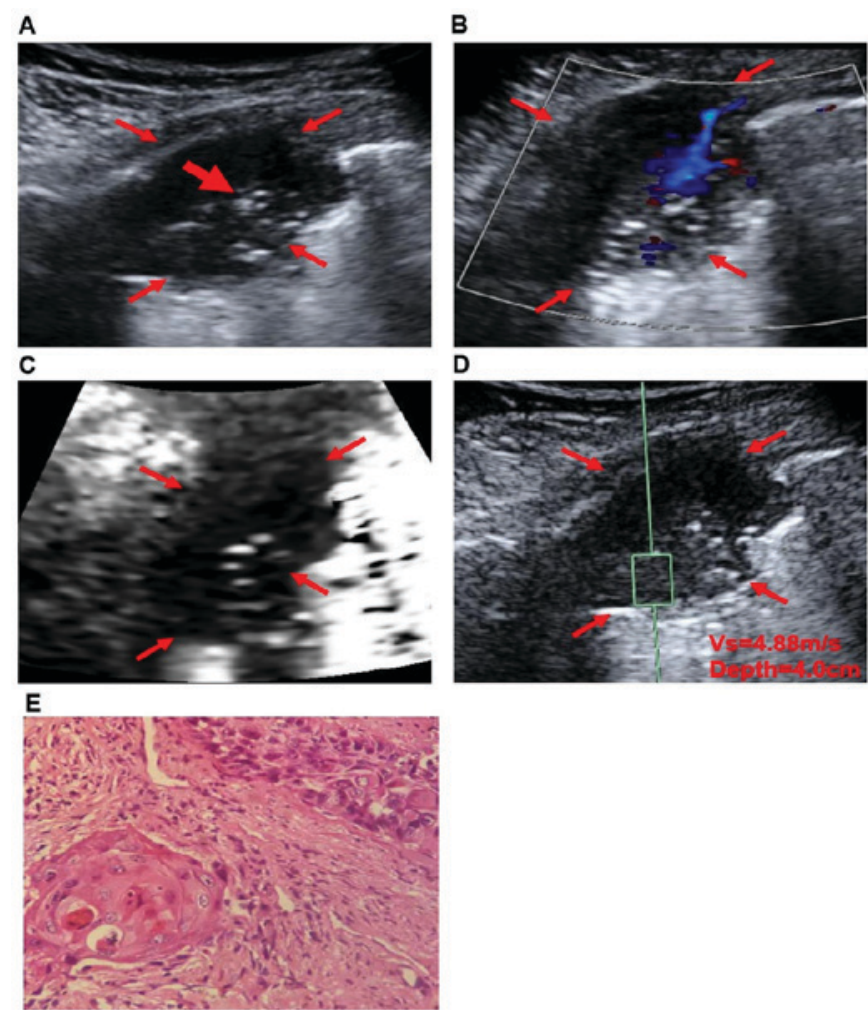

Figure 1. Images of a 64-year-old male with pulmonary squamous cell carcinoma. The arrows in each image indicate the contour of the lesions. (A and B) On conventional ultrasound, a 46- $\mathrm{mm}$ peripheral lung lesion in the right lobe of the lung (arrows in A) appeared to have an irregular shape, a rough margin, hypoechogenicity, heterogeneity, the presence of air bronchogram (the largest arrow in A) and no rare intranodular flow. (C) On acoustic radiation force impulse imaging, the lesion was classified as score 3 (arrows) (D) The share wave elastography of the lesion was $4.88 \mathrm{~m} / \mathrm{sec}$. (E) Histology of the lesion confirmed the diagnosis of squamous cell carcinoma. Hematoxylin and eosin staining; magnification, x100.

0.709 . With a cut-off value of $1.951 \mathrm{~m} / \mathrm{sec}$, the sensitivity and the specificity for diagnosis of malignancy were $70.9 \%(39 / 55)$ and $69.4 \%(25 / 36)$, respectively, with a PPV of $78.0 \%(39 / 50)$ and an NPV of $61.0 \%(25 / 41)$ attached.

\section{Discussion}

US is not a familiar tool for thoracic radiologists due to its infrequent use. However, it still offers several advantages to both the radiologists and patients. Some studies have showed that US guidance is as accurate as CT guidance in obtaining adequate 
Table III. Ultrasound characteristics of benign and malignant peripheral pulmonary lesions.

\begin{tabular}{|c|c|c|c|}
\hline Characteristic & Benign, n (\%) & Malignant, n (\%) & P-value \\
\hline \multicolumn{4}{|c|}{ Diameter of tumor $(\mathrm{cm})$} \\
\hline$<5$ & $14(66.7)$ & $7(33.3)$ & \multirow[t]{2}{*}{$0.006^{\mathrm{b}}$} \\
\hline$\geq 5$ & $22(31.4)$ & $48(68.6)$ & \\
\hline \multicolumn{4}{|l|}{ Shape } \\
\hline Regular contour & $20(51.3)$ & $19(48.7)$ & \multirow[t]{2}{*}{$0.048^{\mathrm{a}}$} \\
\hline Irregular contour & $16(30.8)$ & $36(69.2)$ & \\
\hline \multicolumn{4}{|l|}{ Margin } \\
\hline Smooth & $6(60.0)$ & $4(40.0)$ & \multirow[t]{2}{*}{0.161} \\
\hline Rough & $30(37.0)$ & $51(63.0)$ & \\
\hline \multicolumn{4}{|c|}{ Echogenicity (hypoechoic/hypo-anechoic) } \\
\hline Homogeneous & $6(66.7)$ & $3(33.3)$ & \multirow[t]{2}{*}{0.147} \\
\hline Heterogeneous & $30(36.6)$ & $52(63.4)$ & \\
\hline \multicolumn{4}{|l|}{ Air bronchogram } \\
\hline Absent & $10(26.3)$ & $28(73.7)$ & \multirow[t]{2}{*}{$0.029^{\mathrm{a}}$} \\
\hline Present & $26(49.1)$ & $27(50.9)$ & \\
\hline \multicolumn{4}{|l|}{ Vascularity } \\
\hline Non-abundant & $17(30.9)$ & $38(69.1)$ & \multirow[t]{2}{*}{$0.037^{\mathrm{a}}$} \\
\hline Abundant & $19(52.8)$ & $17(47.2)$ & \\
\hline
\end{tabular}

${ }^{\mathrm{a}} \mathrm{P}<0.05 ;{ }^{\text {b }} \mathrm{P}<0.01$.

Table IV. Ultrasound elastography features of benign and malignant peripheral pulmonary lesions.

\begin{tabular}{lcc}
\hline Elastography features & Benign, n $(\%)$ & Malignant, n $(\%)$ \\
\hline SE-imaging score & & \\
Score 1 & $10(71.4)$ & $4(28.6)$ \\
Score 2 & $17(33.3)$ & $34(66.7)$ \\
Score 3 & $5(29.4)$ & $12(70.6)$ \\
Score 4 & $4(44.4)$ & $5(55.6)$ \\
ARFI-imaging score & & \\
Score 1 & $15(93.8)$ & $1(6.3)$ \\
Score 2 & $4(33.3)$ & $8(66.7)$ \\
Score 3 & $15(37.5)$ & $25(62.5)$ \\
Score 4 & $2(8.7)$ & $21(91.3)$ \\
p-SWE [SWV(m/sec)] & $1.85 \pm 0.92$ & $2.47 \pm 0.92$
\end{tabular}

p-SWE data are presented as the mean \pm standard deviation. ${ }^{a} \mathrm{P}<0.01 ;{ }^{b} \mathrm{P}<0.001$. SE-imaging, strain elastography imaging; ARFI imaging, acoustic radiation force impulse imaging; -SWE, point share wave elastography; SWV, shear wave velocity.

samples from PLLs $(19,20)$. This initial study demonstrated that the diagnostic information of PLLs may be provided by ARFI elastography in addition to radioactive techniques or invasive procedures, while SE was not significant for diagnosis.

Conventional US was capable of detecting lung lesions only when they were located in the peripheral regions of the organ. As there was no typical neoplastic pattern on US, needle biopsy of these lesions was mandatory (21). Sheth et al (22) reported that small PLs appeared hypoechoic and larger PLs were more heterogeneous. However, some studies $(23,24)$ showed that wedge shape, isoechoic or hypoechoic, and air bronchograms might appear on peripheral pulmonary consolidations. In our study, we found that a lesion diameter greater than or equal to $5 \mathrm{~cm}$, irregular contour, presence of air bronchogram, and non-abundant vascularity were predictive factors of malignant PLLs, whereas the margin and echogenicity of lesions were not. An explanation may be that overlapping US characteristics between malignant and benign lesions existed due to the various types of PLL pathologies. In addition, the sensitivity and specificity of US characteristics varied considerably, 30.9-87.3\% and 

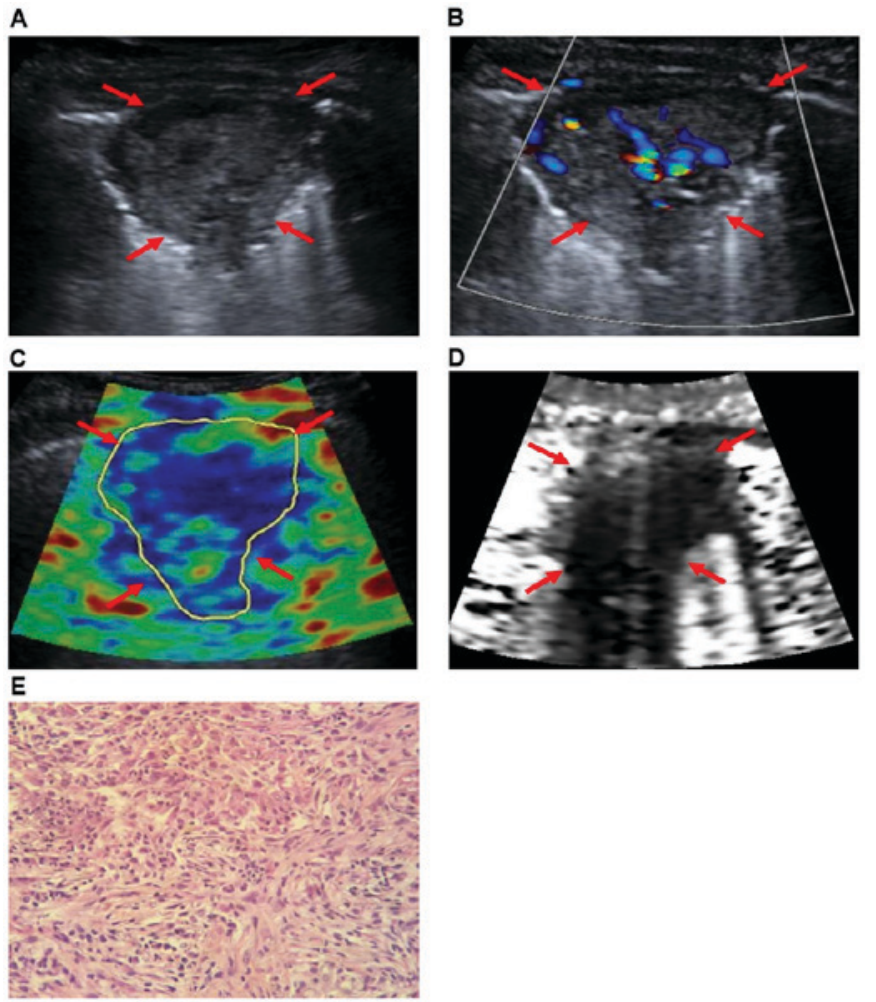

Figure 2. Images of a 40-year-old female with tuberculosis. The arrows in each image indicate the contour of the lesions. (A and B) On conventional ultrasound, a 41-mm peripheral lung lesion in the right lobe of the lung (arrows in A) appeared to have a regular shape, a rough margin, hypoechogenicity, heterogeneity and the presence of rare intranodular flow. (C) On strain elastography imaging, the lesion was predominantly in blue with or without a few green areas and had a score of 4. (D) On acoustic radiation force impulse imaging, the lesion was classified as score 3. (E) Histology of the lesion confirmed the diagnosis of tuberculosis. Hematoxylin and eosin staining; magnification, x100.

$16.7-55.6 \%$, respectively, and the results were not satisfactory. Therefore, additional methods should be adopted to diagnose PLLs.

SE was introduced to assess the tissue stiffness by hand or using cardiovascular pulsation or respiratory motion to achieve compression. However, some studies revealed that it was difficult for radiologists to control the pressure using manual compression in SE examination, thus leading to considerably low reproducibility $(25,26)$. In our study, we also found the same problem due to the limitation of cardiovascular pulsation or respiratory motion, which made the compression inconsistent. Furthermore, no significant differences on SE were found between BLLs and MLLs. In the study by Sperandeo et al (21), which performed TUS elastography on 95 patients with PLs, only squamous cell lung carcinoma displayed increased stiffness. In their study, TUS elastography was limited in characterizing lesions, which was similar to our result. Additionally, 1 lesion with a score of 2 proved to be adenocarcinoma; a possible pathological explanation might be that the cells contained many acini and had intracellular mucus.

To the best of our knowledge, this research was the first preliminary study to detect the diagnostic performance of ARFI imaging and p-SWE in PLLs. ARFI elastography was more effective in diagnosing PLLs, with the advantages of free-hand compression and semiquantitative or quantitative measurements. In our study, the ARFI score of the malignant PLLs was higher than that of the benign ones $(\mathrm{P}=0.011)$, indicating higher stiffness. When score 3 was applied as cut-off value, the corresponding sensitivity was $83.6 \%(46 / 55)$, which was higher than chest radiography (73.5\%) (27). Thus, a malignant lesion may be suggested before surgery on the condition that it obtained a score of 3 or more on ARFI imaging. Under such circumstances, we found that tuberculosis (a total of 4 cases) had a score of 3 and 1 chronic inflammation had a score of 4 on ARFI imaging. This phenomenon might be explained by the formation of lung fibrous band structures in tuberculosis and the proliferation of fibrosis in chronic inflammation, which might increase the tissue stiffness (Fig. 2). However, our study was limited by a small sample size; there is a need for a separate study with more tuberculosis and chronic pneumonia patients for further analysis.

Compared to SE, p-SWE was applied to evaluate the tissue stiffness by generating shear wave, with the advantages of a smaller subjective influence of the operator, independent elastic image quality, and quantitative measurement. In our study, the mean SWV values of the malignant lesions were higher than those of the benign lesions $(2.47 \pm 0.92$ vs. $1.85 \pm 0.92 \mathrm{~m} / \mathrm{sec}$; $\mathrm{P}=0.002$ ). With a cut-off value of $1.951 \mathrm{~m} / \mathrm{sec}$, the sensitivity, specificity, PPV, and NPV for diagnosing MLLs were 70.91, $69.44,78.00$, and $60.98 \%$, respectively. It was observed that p-SWE was more effective than SE in distinguishing malignant from benign lesions. Although the sensitivity of p-SWE was not as good as low-dose CT, their specificity was similar (73.4\%). Furthermore, the specificity of p-SWE was not as good as that of chest radiography, but the sensitivity was similar (27). s P-SWE has provided a new method for evaluating the hardness of PLLs. However, false positives and false negatives also existed in p-SWE examination. Among the benign lesions, 1 chronic inflammatory lesion had a mean SWV of $4.344 \mathrm{~m} / \mathrm{sec}$, which may indicate the existence of fibrosis in the lesion. In addition, 1 small cell lung cancer had a mean SWV of $1.202 \mathrm{~m} / \mathrm{sec}$ and an ARFI imaging score of 2, which might be explained by the complex heterogeneity of tumor cells (28).

There were some limitations in this study. First, the selection bias was unavoidable due to its retrospective nature. Second, the inter-observer and intra-observer agreements were not analyzed, although some studies have shown that ARFI elastography has good reproducibility in multiple organs of the body $(29,30)$. Third, considering the small sample size of our study, prospective research on a large sample is necessary.

In conclusion, our study indicated that US, especially ultrasound elastography, is able to provide doctors with identification methods of PLLs.

\section{Acknowledgements}

Not applicable.

\section{Funding}

The present study was funded by the Scientific Research of Heilongjiang Province Health and Family Planning Commission (grant no. 2014-334). 


\section{Availability of data and materials}

The datasets used and/or analyzed during the current study are available from the corresponding author on reasonable request.

\section{Authors' contributions}

HW was a major contributor in performing the experiments and writing the manuscript. YL collated and analyzed the ultrasonic images. QJ conducted the data analysis, and HZ analyzed the pathological results. XZ led this study, performed the pathological biopsies of the lungs and gave final approval of the version to be published. All authors read and approved the final manuscript.

\section{Ethics approval and consent to participate}

This retrospective study was approved by the Ethics Committee of this tertiary hospital (the Second Affiliated Hospital of Harbin Medical University, Harbin, China) and the written informed consent of patients was obtained.

\section{Patient consent for publication}

Informed consent was obtained from all individual participants included in this study.

\section{Competing interests}

The authors declare that they have no competing interests.

\section{References}

1. Goozner M: A tale of two countries: Lung cancer care in Brazil and China. J Natl Cancer Inst 104: 1621-1623, 2012.

2. Kim CH, Lee YC, Hung RJ, McNallan SR, Cote ML, Lim WY, Chang SC, Kim JH, Ugolini D, Chen Y, et al: Exposure to secondhand tobacco smoke and lung cancer by histological type: A pooled analysis of the International Lung Cancer Consortium (ILCCO). Int J Cancer 135: 1918-1930, 2014.

3. Chen W, Liu Y, Wang H, Hnizdo E, Sun Y, Su L, Zhang X, Weng S, Bochmann F, Hearl FJ, et al: Long-term exposure to silica dust and risk of total and cause-specific mortality in Chinese workers: A cohort study. PLoS Med 9: e1001206, 2012.

4. Strauss GM, Gleason RE and Sugarbaker DJ: Chest X-ray screening improves outcome in lung cancer. A reappraisal of randomized trials on lung cancer screening. Chest 107 (6 Suppl): 270S-279S, 1995.

5. Yankelevitz DF: Point: Should lung cancer screening by chest CT scan be a covered benefit? Yes. Chest 147: 287-289, 2015.

6. Field JK, Devaraj A, Duffy SW and Baldwin DR: CT screening for lung cancer: Is the evidence strong enough? Lung Cancer 91: 29-35, 2016.

7. Adamietz BR, Fasching PA, Jud S, Schulz-Wendtland R, Anders K, Uder M, Wüst W, Rauh C and Meier-Meitinger M: Ultrasound elastography of pulmonary lesions-a feasibility study. Ultraschall Med 35: 33-37, 2014.

8. Volpicelli G: Lung sonography. J Ultrasound Med 32: 165-171, 2013.

9. Sconfienza LM, Mauri G, Grossi F, Truini M, Serafini G, Sardanelli F and Murolo C: Pleural and peripheral lung lesions: Comparison of US- and CT-guided biopsy. Radiology 266: 930-935, 2013.

10. Rhymer JC: Elastography in the detection of prostatic cancer. Clin Radiol 58: 337, 2003.

11. Xu JM, Xu XH, Xu HX, Zhang YF, Zhang J, Guo LH, Liu LN, Liu $\mathrm{C}$ and Zheng SG: Conventional US, US elasticity imaging, and acoustic radiation force impulse imaging for prediction of malignancy in thyroid nodules. Radiology 272: 577-586, 2014.
12. Guo LH, Wang SJ, Xu HX, Sun LP, Zhang YF, Xu JM, Wu J, $\mathrm{Fu} \mathrm{HJ}$ and $\mathrm{Xu} \mathrm{XH}$ : Differentiation of benign and malignant focal liver lesions: Value of virtual touch tissue quantification of acoustic radiation force impulse elastography. Med Oncol 32: $68,2015$.

13. Ko KH, Jung HK, Kim SJ, Kim H and Yoon JH: Potential role of shear-wave ultrasound elastography for the differential diagnosis of breast non-mass lesions: Preliminary report. Eur Radiol 24: 305-311, 2014.

14. Cross TJ, Mitchell JD and Cramp ME: Elastography for the non-invasive assessment of liver disease: Limitations and future developments. Gut 58: 1171-1172, 2009.

15. Moon HJ, Sung JM, Kim EK, Yoon JH, Youk JH and Kwak JY: Diagnostic performance of gray-scale US and elastography in solid thyroid nodules. Radiology 262: 1002-1013, 2012.

16. Ophir J, Céspedes I, Ponnekanti H, Yazdi Y and Li X: Elastography: A quantitative method for imaging the elasticity of biological tissues. Ultrason Imaging 13: 111-134, 1991.

17. Shiina T, Nightingale KR, Palmeri ML, Hall TJ, Bamber JC, Barr RG, Castera L, Choi BI, Chou YH, Cosgrove D, et al: WFUMB guidelines and recommendations for clinical use of ultrasound elastography: Part 1: Basic principles and terminology. Ultrasound Med Biol 41: 1126-1147, 2015.

18. Sperandeo M, Trovato FM, Dimitri L, Catalano D, Simeone A, Martines GF, Piscitelli AP and Trovato GM: Lung transthoracic ultrasound elastography imaging and guided biopsies of subpleural cancer: A preliminary report. Acta Radiol 56: 798-805, 2015.

19. Lichtenstein D, Mezière G and Seitz J: The dynamic air bronchogram. A lung ultrasound sign of alveolar consolidation ruling out atelectasis. Chest 135: 1421-1425, 2009.

20. Jeon KN, Bae K, Park MJ, Choi HC, Shin HS, Shin S, Kim HC and Ha CY: US-guided transthoracic biopsy of peripheral lung lesions: Pleural contact length influences diagnostic yield. Acta Radiol 55: 295-301, 2014.

21. Sperandeo M, Filabozzi P, Varriale A, Carnevale V, Piattelli ML, Sperandeo G, Brunetti E and Decuzzi M: Role of thoracic ultrasound in the assessment of pleural and pulmonary diseases. J Ultrasound 11: 39-46, 2008.

22. Sheth S, Hamper UM, Stanley DB, Wheeler JH and Smith PA: US guidance for thoracic biopsy: A valuable alternative to CT. Radiology 210: 721-726, 1999.

23. Yang PC, Luh KT, Chang DB, Yu CJ, Kuo SH and Wu HD: Ultrasonographic evaluation of pulmonary consolidation. Am Rev Respir Dis 146: 757-762, 1992.

24. Yang PC, Chang DB, Yu CJ, Lee YC, Kuo SH and Luh KT: Ultrasound guided percutaneous cutting biopsy for the diagnosis of pulmonary consolidations of unknown aetiology. Thorax 47: 457-460, 1992.

25. Kim MH, Luo S, Ko SH, Bae JS, Lim J, Lim DJ and Kim Y: Thyroid nodule parameters influencing performance of ultrasound elastography using intrinsic compression. Ultrasound Med Biol 41: 2333-2339, 2015.

26. Chang JM, Won JK, Lee KB, Park IA, Yi A and Moon WK: Comparison of shear-wave and strain ultrasound elastography in the differentiation of benign and malignant breast lesions. AJR Am J Roentgenol 201: W347-W356, 2013.

27. National Lung Screening Trial Research Team, Church TR, Black WC, Aberle DR, Berg CD, Clingan KL, Duan F, Fagerstrom RM, Gareen IF, Gierada DS, et al: Results of initial low-dose computed tomographic screening for lung cancer. N Engl J Med 368: 1980-1991, 2013.

28. Krohn A, Ahrens T, Yalcin A, Plönes T, Wehrle J, Taromi S, Wollner S, Follo M, Brabletz T, Mani SA, et al: Tumor cell heterogeneity in Small Cell Lung Cancer (SCLC): Phenotypical and functional differences associated with Epithelial-Mesenchymal Transition (EMT) and DNA methylation changes. PLoS One 9: e100249, 2014.

29. Ma JJ, Ding H, Mao F, Sun HC, Xu C and Wang WP: Assessment of liver fibrosis with elastography point quantification technique in chronic hepatitis B virus patients: A comparison with liver pathological results. J Gastroenterol Hepatol 29: 814-819, 2014.

30. Bob F, Bota S, Sporea I, Sirli R, Petrica L and Schiller A: Kidney shear wave speed values in subjects with and without renal pathology and inter-operator reproducibility of acoustic radiation force impulse elastography (ARFI)-preliminary results. PLoS One 9: e113761, 2014.

This work is licensed under a Creative Commons Attribution-NonCommercial-NoDerivatives 4.0 International (CC BY-NC-ND 4.0) License. 